\title{
DETERMINAÇÃO DO PADRÃO DE OPERAÇÃO ÓTIMO PARA O CUSTO ENERGÉTICO DE UM SISTEMA DE DISTRIBUIÇÃO DE ÁGUA
}

\section{OPTIMIZATION OF PUMP OPERATION PATTERNS FOR REDUCING THE ENERGETIC COST OF A WATER DISTRIBUTION SYSTEM}

\author{
Marina Bouzon* E-mail: marinabouzon@gmail.com \\ Antônio Sérgio Coelho* E-mail: coelho@deps.ufsc.br \\ Carlos M. Taboada Rodriguez* E-mail: taboada@deps.ufsc.br \\ *Universidade Federal de Santa Catarina, Florianópolis, SC
}

\begin{abstract}
Resumo: O manejo otimizado dos sistemas de abastecimento de água é um fator essencial para o bem estar da sociedade. Neste contexto, as empresas de saneamento enfrentam um grande desafio na operação dos sistemas de bombeamento de água para abastecimento hídrico das cidades: determinar a utilização ótima de seus recursos a fim de minimizar o seu custo energético (CE) de operação. Este trabalho apresenta um modelo de otimização em Programação Linear (PL) para o funcionamento de um sistema de distribuição de água de uma cidade no interior do Estado de São Paulo, que contempla duas bombas e três reservatórios de água. Foi criado um modelo para determinar o padrão ótimo de operação para as duas bombas no período de 24 horas, a fim de minimizar o custo energético e, ao mesmo tempo, obedecer às restrições de capacidade de reservação e atendimento da demanda. Os resultados obtidos neste trabalho mostram que a utilização da PL é satisfatória para a resolução deste tipo de problema, podendo beneficiar a empresa de saneamento, a população atendida e até mesmo o meio-ambiente, ao consumir menos energia.
\end{abstract}

Palavras-chave: Distribuição de água. Programação linear. Custo energético.

Abstract: The optimal management of water supply systems is a critical factor for the well-being of a society. In this context, water companies face a major challenge in the operation of water pumping systems for water supply of cities: to determine the optimal use of its resources in order to minimize the energy cost $(E C)$ of operation. This paper presents an optimization model in Linear Programming (LP) for the operation of a water distribution system in a city of the São Paulo State comprising two pumps and three water tanks. A model was created to determine the optimal pattern of operation for the two bombs within 24 hours in order to minimize the EC and at the same time, comply with the capacity reservation constraints and meet demand. The results of this study show that the use of PL may be satisfactory for the resolution of this problem and can provide benefits for the water company, the population served and for the environment by improving the energetic efficiency of a water distribution system.

Key-words: Water distribution. Linear programming. Energy cost. 


\section{INTRODUÇÃO}

De acordo com o Programa Nacional de Conservação de Energia Elétrica para o Saneamento (SANEAR, 2006), o bombeamento e tratamento de água e esgoto representam mais de $2 \%$ do consumo de energia elétrica no Brasil, 0 equivalente a sete bilhões de kWh/ano. Deste valor, estima-se que mais de $90 \%$ deve-se às elevatórias dos sistemas de abastecimento de água (TSUTIYA, 2001).

Em paralelo a este dado, afirma-se que a utilização de recursos de análises de sistemas, tais como ferramentas computacionais de otimização, pode trazer economias de até $30 \%$ para grandes empreendimentos, o que representaria dezenas ou centenas de milhões de dólares (FRANCATO; BARBOSA, 1999).

Desta maneira, faz-se importante a aplicação de ferramentas de otimização com uso de modelagem matemática em sistemas de bombeamento de água, a fim de minimizar os gastos e, ao mesmo tempo, atender às exigências dos sistemas, tais como: restrições hidráulicas, atendimento da demanda de água e manutenção de pressões adequadas. Para isso, é necessária a determinação das relações lógico-matemáticas do sistema hídrico, a fim de transformar este problema físico em um problema matemático.

Neste contexto, o objetivo desta pesquisa foi desenvolver um modelo de otimização que considera as restrições hidráulicas e ao mesmo tempo a demanda do sistema com foco na determinação do padrão ótimo de funcionamento das bombas, a fim de minimizar o custo energético. Desta maneira, este artigo apresenta um algoritmo para otimização em Programação Linear para minimização de custos energéticos em um sistema de abastecimento de água em uma cidade no interior do Estado de São Paulo. A análise foi realizada sob um determinado esquema tarifário da concessionária de energia elétrica, que varia de acordo com o período do dia utilizado. Além disso, a otimização de custo visou obedecer às restrições do sistema, tais como vazão de fornecimento de água da Estação de Tratamento de Água (ETA), capacidades mínima e máxima dos reservatórios, demanda de água do sistema, dentre outras. A solução do problema forneceu um padrão de funcionamento para cada uma das bombas em termos de tempo de bomba ligada para cada hora do dia, variando de zero a 60 minutos. 


\section{FUNDAMENTAÇÃO TEÓRICA}

Um sistema de distribuição de água é uma rede de componentes (e.g. tubulações, bombas, válvulas, tanques, etc.) que transporta água de uma fonte (e.g. reservatório, estação de tratamento, tanque, etc.) até os consumidores (e.g. usuário doméstico, comercial e industrial) (ZECCHIN et al., 2006).

O objetivo da operação de sistemas de distribuição de água é atender, de maneira confiável, as necessidades de consumo, a custos operacionais mínimos, tais como os de consumo de energia e manutenção. Por isso, pode-se afirmar que o trabalho no setor de operações em um sistema de abastecimento requer decisões eficientes, conforme regra operacional adequada. Para otimizar esta regra, é necessário ter conhecimento de alguns parâmetros do sistema, tais como: definição clara dos objetivos a serem alcançados, conhecimento do sistema e dos dados previsionais e disponibilidade de um modelo para otimizar essas regras (CUNHA, 2009).

Devido ao alto custo associado com a construção e operação de sistemas de distribuição de água, muitas pesquisas nos últimos 25 anos foram dedicadas a desenvolver técnicas para minimizar custos (ZECCHIN et al., 2006). Nas últimas três décadas, muitos estudos foram dedicados para projetar e operar sistemas de distribuição de água ótimos usando diversos algoritmos de otimização (KANG; LANSEY, 2011).

As técnicas de Pesquisa Operacional (PO) vêm ganhando importância na análise de sistemas hídricos, uma vez que viabilizam uma modelagem mais adequada de problemas reais. Neste sentido, algumas técnicas vêm sendo utilizadas para solução de problemas nesta área, tais como: programação linear, programação dinâmica, otimização de fluxo em redes (FRANCATO; BARBOSA, 1999), redes neurais artificiais (RNA) e algoritmos genéticos (CUNHA, 2009). Segundo Yang et al. (1996), entre os trabalhos realizados com aplicação de PO em sistemas hídricos, há uma preferência pela programação linear por propiciar algumas vantagens, como: adequação para problemas de grandes dimensões, não necessidade de uma política inicial e grande número de sub-rotinas já disponíveis. 
Um modelo de Programação Linear é um modelo matemático de otimização no qual todas as funções são lineares (GOLDBARG; LUNA, 2000).

Conforme Francato e Barbosa (1999), na literatura existente, é possível encontrar várias experiências bem sucedidas com a aplicação de técnicas de PO na tentativa de redução dos custos operacionais em sistemas de distribuição de água. Os algoritmos podem visar diferentes objetivos a serem alcançados, como a redução da vazão bombeada, associada às despesas com energia elétrica, que corresponde ao principal custo na operação de um sistema de abastecimento (VENTURINI, 1997).

Para reduzir o consumo de energia elétrica de sistemas de abastecimento de água, algumas alternativas podem ser tomadas, tais como (TSUTIYA, 2001): i) conhecimento do sistema tarifário; ii) redução da potência do equipamento; iii) alteração do sistema operacional; iv) automação do sistema de abastecimento de água; v) geração de energia via fontes alternativas de energia elétrica.

Em geral, a maior parte do custo energético é associada à operação de bombas. Por isso, um padrão ótimo de operação de bombas pode reduzir os custos significativamente enquanto mantém as necessidades de operação do sistema (PASHA; LANSEY, 2009), como o atendimento da demanda e as restrições de capacidade e pressão. Nesse sentido, o foco deste estudo é a redução de custo de energia elétrica utilizada por meio de otimização do padrão ou regra de operação das bombas.

\section{METODOLOGIA}

Este tópico reúne as etapas da metodologia utilizada para a solução do problema de abastecimento de água. A etapa inicial do trabalho foi a coleta dos dados do sistema de abastecimento de água para montagem do esquema hídrico e determinação do escopo da pesquisa. Os dados foram obtidos diretamente com uma empresa terceirizada de Engenharia e Soluções Tecnológicas que presta serviços para a empresa de distribuição de água. As informações foram obtidas de banco de dados com histórico de operação do sistema, assim como planilhas com dados de capacidade e cota dos reservatórios, potência e vazão das bombas. 
A metodologia aplicada neste trabalho é a Programação Linear ( $P L)$. Um modelo de programação linear tem como função objetivo (FO) minimizar ou maximizar um valor " $z$ ". Esta função é sujeita a várias restrições de recursos, e todas as equações do modelo são equações lineares (DANTZIG, 1998).

\subsection{Montagem do problema}

Para modelar o presente problema através da $\mathrm{PL}$, os seguintes passos foram adotados (GOLDBARG; LUNA, 2000):

- Escolha da variável de decisão: custo energético (CE);

- Elaboração da função objetivo (FO): minimizar CE;

- Formulação das restrições tecnológicas: detalhadas no item 5.3;

- Restrições de não-negatividade.

\subsection{Utilização de software para solucionar o PPL}

Diversos softwares como o LINGO, LINDO, CPLEX, MOSEK e muitos outros solucionam modelos através dos algoritmos-padrão (COSTA; SILVA, 2010). O software utilizado para solucionar o problema de otimização do custo de distribuição é o LINDO (Linear, INteractive, and Discrete Optimizer). O LINDO é uma conveniente e poderosa ferramenta para resolver problemas de Programação Linear, inteira e quadrática. Um modelo do LINDO deverá conter: uma função objetivo; seguida por uma declaração "subject to" (sujeito a) ou simplesmente usa-se "st", logo após são declaradas as restrições do problema; e deve ser finalizado com o comando "end" (GOMES JR; SOUZA, 2004).

Esta ferramenta ainda permite analisar a sensibilidade do modelo criado, o que significa saber o quanto pode ser alterado um ou mais parâmetros do modelo (coeficientes, recursos, etc.) e ainda assim manter a mesma solução ótima. 


\section{DESCRIÇÃO DO PROBLEMA}

Uma rede hidráulica, em geral, é constituída por vários elementos, cada um com sua determinada função. São elementos comuns encontrados em sistemas de abastecimento de água: tubulações, reservatórios, bombas e válvulas. Um sistema de abastecimento de água, em geral, engloba a captação de água bruta de um determinado manancial, que segue por adutoras até uma Estação de Tratamento de Água (ETA). Da ETA, a água tratada é bombeada para reservatórios que se localizam acima do nível da cidade e/ou bairros a serem abastecidos.

O município do estudo de caso está situado na região sudoeste do Estado de São Paulo, sendo que $100 \%$ da população urbana é atendida pelo sistema de abastecimento de água. Por questões de confidencialidade de dados, a empresa de distribuição de água estudada será chamada de "Empresa A".

O objetivo principal da Empresa A engloba a captação, adução, tratamento, recalque e distribuição de água potável à população do município, assim como a coleta, tratamento e afastamento do esgoto sanitário gerado na cidade.

A Empresa A opera hoje com quatro ETAs, uma Estação de Reservação e Distribuição e três poços profundos. A ETA IV possui capacidade de tratamento de até $12 \mathrm{~L} / \mathrm{s}$, e reservação de $200 \mathrm{~m}^{3}$. É responsável por abastecer dois bairros do município, sem interligação hidráulica com os demais sistemas de abastecimento da cidade.

Além da estação de tratamento em si, este sistema hidráulico possui duas estações elevatórias de água; a primeira, anexa à ETA, é responsável pelo recalque de água até a Estação Elevatória de Água Tratada (EAT); nesta a água é bombeada para um reservatório elevado (REL) localizado na própria estação, a partir do qual é distribuída para a população dos bairros.

Assim, além da ETA, este sistema possui duas bombas que operam em potência fixa e três reservatórios de água tratada, conforme ilustra a Figura 1 (para análise deste sistema, bombas reserva não foram consideradas). 
Figura 1 - Esquema das estações elevatórias de água tratada.

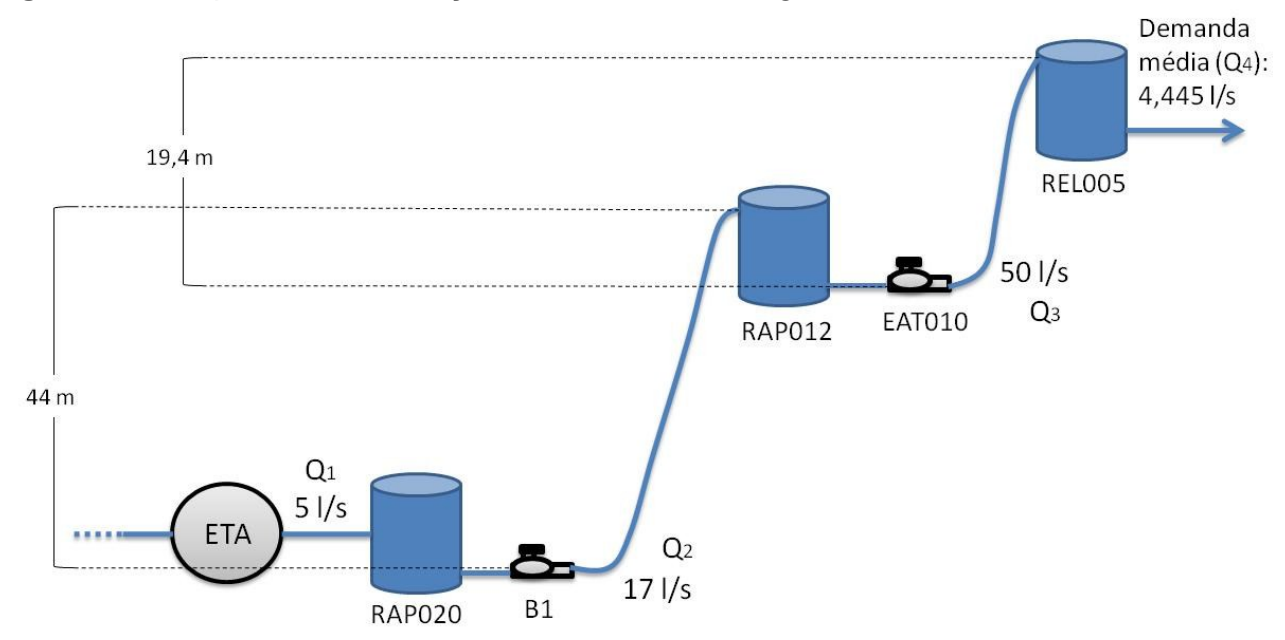

A água é tratada pela ETA, sendo que a vazão média que chega ao primeiro reservatório, chamado RAP020, é de 5 litros por segundo. Deste reservatório, a bomba B1 funciona a uma potência hidráulica de 8,333 kW, pressão de operação 50 metros de coluna d'água (mca) e vazão de operação 17 L/s. A pressão e vazão nominal são diferentes, 45 mca e $19 \mathrm{~L} / \mathrm{s}$, respectivamente. Isto ocorre em função da perda de carga associada à tubulação do sistema, o que gera valores reais (de operação) diferentes. Desta maneira, serão considerados os valores de operação para o problema.

A água chega ao reservatório RAP012 com vazão de $17 \mathrm{~L} / \mathrm{s}$, onde fica armazenada até ser enviada para o reservatório de elevação REL005 pela bomba EAT010. Esta bomba funciona a uma potência hidráulica de operação de 12,75 kW, com vazão de 50 L/s e pressão de 26 mca (valores operacionais). A partir do reservatório elevado REL005 a água abastece o bairro por pressão hidráulica de diferença de cota.

As bombas são componentes hidráulicos usados nos sistemas de distribuição de água com a finalidade de manter as vazões requeridas com altura manométrica adequada, viabilizando a operação do sistema (VENTURINI, 1997). Ambas as bombas funcionam em uma potência constante durante o dia, e podem estar ligadas ou desligadas, conforme necessidades do sistema, e possuem rendimento de $75 \%$ $(\eta=0,75)$.

Para este trabalho, a pressão relativa ao nível do reservatório na sucção da bomba foi desconsiderada para efeito de cálculo da potência hidráulica. A adutora 
de entrada dos três reservatórios é localizada na parte superior dos mesmos, ou seja, acima do nível máximo de operação dos reservatórios. Em consequência disso, o nível de água no reservatório não influencia diretamente a pressão de recalque das bombas. Os dados dos reservatórios do sistema encontram-se na Tabela 1.

Tabela 1 - Dados dimensionais dos reservatórios.

\begin{tabular}{lccccc}
\hline Reservatório & Diâmetro $(\mathbf{m})$ & Altura útil $(\mathbf{m})$ & Volume máx. $\left(\mathbf{m}^{\mathbf{3}}\right)$ & Volume $\mathbf{m i ́ n .}\left(\mathbf{m}^{\mathbf{3}}\right)$ & Volume inicial $\left(\mathbf{m}^{\mathbf{3}}\right)$ \\
\hline RAP020 & 4,426 & 6,500 & 100,028 & 33,343 & 70,020 \\
RAP012 & 7,640 & 8,800 & 403,422 & 134,474 & 282,395 \\
REL005 & 4,450 & 6,700 & 104,204 & 34,735 & 72,943 \\
\hline
\end{tabular}

Fonte: Dados da Empresa A.

O custo operacional de um sistema de distribuição de água é basicamente o consumo de energia elétrica, em kWh, consumida durante o período de faturamento. O tarifário da concessionária de energia elétrica adotado é o horo-sazonal verde, o qual opera com duas faixas de preços, de acordo com a hora do dia, conforme Tabela 2 (foram desconsiderados custos tarifários relativos à demanda contratada).

Tabela 2 - Tarifário horo-sazonal verde para saneamento.

\begin{tabular}{lcc}
\hline Unidade & Hora de Ponta (18:00 as 20:59) $-\mathrm{T}_{\mathbf{2}}$ & Hora Fora de Ponta (demais períodos) $-\mathrm{T}_{\mathbf{1}}$ \\
\hline $\mathrm{R} \$ / \mathrm{MWh}$ & 749,088 & 140,93 \\
$\mathrm{R} \$ / \mathrm{kWh}$ & 0,7491 & 0,1409 \\
\hline
\end{tabular}

Fonte: Dados da concessionária de energia elétrica.

É importante mencionar que as companhias de serviço de água e esgoto recebem $15 \%$ de desconto sobre o valor da concessionária, desconto este já incluso nos valores da Tabela 2 .

A demanda do sistema em questão oscila durante as horas do dia, apresentando dois horários de pico de consumo e um padrão diário conforme dados da Tabela 3 e Gráfico 1. 
Tabela 3 - Padrão de consumo de água em cada intervalo de hora do dia.

\begin{tabular}{|c|c|c|c|c|c|c|c|c|c|c|c|c|}
\hline Hora & 1 & 2 & 3 & 4 & 5 & 6 & 7 & 8 & 9 & 10 & 11 & 12 \\
\hline Vazão (I/s) & 1,689 & 1,600 & 1,378 & 1,200 & 1,200 & 1,422 & 3,867 & 4,534 & 5,823 & 6,579 & 8,801 & 7,557 \\
\hline Vazão (m³) & 6,081 & 5,761 & 4,961 & 4,321 & 4,321 & 5,121 & 13,922 & 16,322 & 20,963 & 23,683 & 31,684 & 27,203 \\
\hline Hora & 13 & 14 & 15 & 16 & 17 & 18 & 19 & 20 & 21 & 22 & 23 & 24 \\
\hline Vazão (I/s) & 6,890 & 6,756 & 5,467 & 5,823 & 5,423 & 4,445 & 6,534 & 5,734 & 4,534 & 4,134 & 2,756 & 2,578 \\
\hline Vazão (m³ $/ \mathrm{h})$ & 24,803 & 24,323 & 19,682 & 20,963 & 19,522 & 16,002 & 23,523 & 20,643 & 16,322 & 14,882 & 9,921 & 9,281 \\
\hline
\end{tabular}

Gráfico 1 - Comportamento padrão de consumo (vazão) em cada intervalo de hora do dia

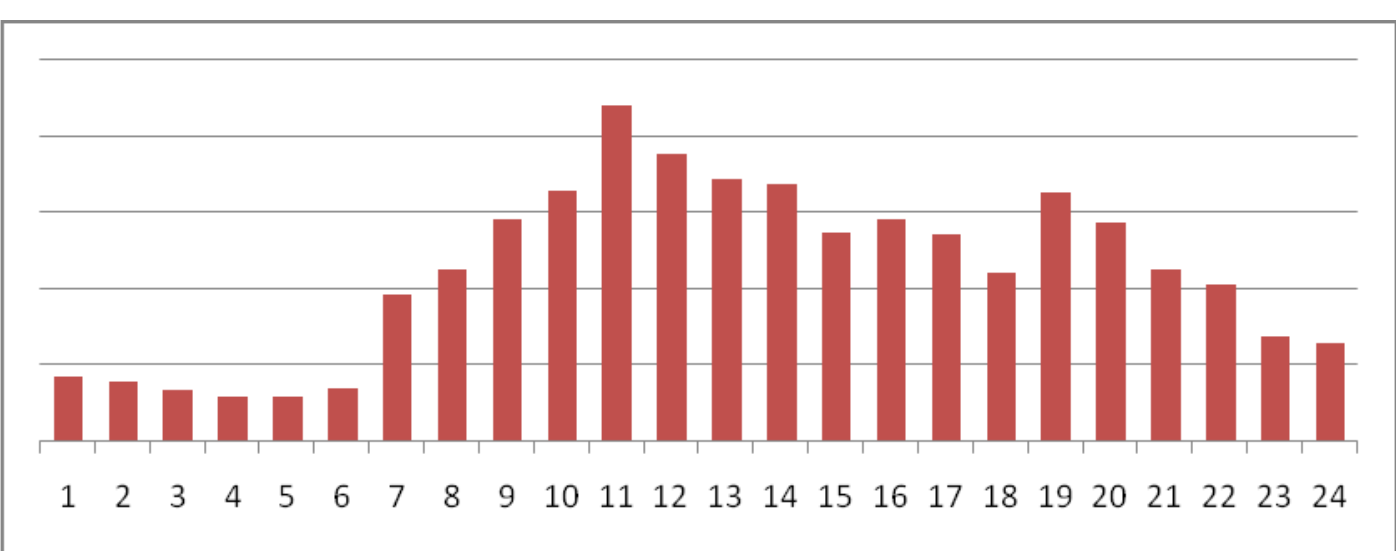

Fonte: Dados da Empresa A.

Com os dados apresentados, o próximo passo é formular o problema de abastecimento em um modelo matemático.

\section{FORMULAÇÃO MATEMÁTICA DO PROBLEMA}

Este tópico aborda passo a passo a montagem do modelo matemático segundo a PL. É considerado o sistema de análise a partir do reservatório RAP020, o que significa que os gastos com energia referentes ao tratamento de água não foram considerados.

Revista Produção Online, Florianópolis, SC, v.13, n. 2, p. 500-519, abr./jun. 2013. 


\subsection{Escolha da Variável de decisão}

Como o objetivo é minimizar os custos energéticos do sistema associados ao consumo elétrico das bombas, a variável de decisão escolhida é aquela que altera o custo de energia. Neste caso é o tempo que cada bomba fica ligada durante um dia, que implicará no consumo de KWh. Será adotado que a potência das bombas é fixa, ou seja, ou está desligada, sem consumo, ou ligada consumindo energia a uma taxa fixa. Desta forma, a notação da variável será:

$$
t_{b i}=\text { tempo de operação da bomba "b" na hora "i" (em horas) }
$$

Sendo que " $b$ " pode assumir B1 ou EAT (bombas do sistema), ou seja: b = B1 ou EAT; e $0 \leq \mathrm{i} \leq 24$, i $\in \mathrm{N}$.

\subsection{Elaboração da Função Objetivo}

O objetivo a ser alcançado é minimizar os custos energéticos do sistema. Por isso, busca-se como resultado da modelagem a determinação de um plano de ação ótimo para as bombas no decorrer das 24 horas de um dia, com o menor custo.

$$
\mathrm{F}_{\mathrm{obj}} \rightarrow \text { minimizar Custo Energético (CE) do sistema com duas bombas }
$$

O custo energético deste sistema é dado pela multiplicação da potência energética pelo tempo de uso (bomba ligada) e pela tarifa da concessionária.

Sendo o CE:

$$
C E=P_{\theta} \cdot t \cdot T
$$

$\mathrm{E}:$

$$
P_{e}=\frac{P_{h}}{\eta}
$$

$\mathrm{E}:$

$$
P_{h}=Q \cdot p
$$

Onde:

$P_{\mathrm{e}}$ : $\quad$ Potência energética

$P_{h}$ : Potência hidráulica

$\eta$ : $\quad$ Rendimento da bomba (constante adimensional $=0,75$ ) 


\section{Q: Vazão \\ p: Pressão \\ t: $\quad$ Tempo de operação da bomba \\ T: $\quad$ Tarifa}

Assim, para o CE total do sistema da Empresa A, deve ser considerado o consumo das duas bombas (B1 e EAT), assim como diferenciado os horários de uso devido às duas tarifas de energia da concessionária $\left(T_{1}\right.$ e $\left.T_{2}\right)$.

Desta forma, a FO pode ser representada por:

$$
\begin{aligned}
\operatorname{Min} C E=P_{\theta B 1} & \cdot\left(\sum_{i=1}^{18} t_{B 1 i} \cdot T_{1}+\sum_{i=19}^{21} t_{B 1 i} \cdot T_{2}+\sum_{i=22}^{24} t_{B 1 i} \cdot T_{1}\right) \\
& +P_{E E A T} \cdot\left(\sum_{i=1}^{18} t_{E A T i} \cdot T_{1}+\sum_{i=19}^{21} t_{E A T i} \cdot T_{2}+\sum_{i=22}^{24} t_{E A T i} \cdot T_{1}\right)
\end{aligned}
$$

Sendo:

CE: Custo Energético de operação em um dia $(R \$)$

$P_{\mathrm{eB} 1}$ : Potência energética da Bomba B1 $(\mathrm{kWh})$

$P_{\text {eEAT: }}$ Potência energética da Bomba EAT010 (kWh)

$t_{B 11}$ : tempo de operação da bomba B1 da hora "i-1" até a hora "i" $(\mathrm{h})$

tEAT: : tempo de operação da bomba EAT010 da hora "i-1" até a hora "i" (h)

$T_{1}$ : Tarifa de operação de horário fora de ponta $(R \$ / k W h)$

$T_{2}$ : Tarifa de operação de horário de ponta $(R \$ / k W h)$

\subsection{Formulação das restrições tecnológicas}

a) Conjunto de restrições para o tempo máximo de utilização da bomba no decorrer do dia, por hora. Esta restrição garante que a solução a ser encontrada não apontará funcionamento de bomba por hora superior a 60 minutos.

(a.1) $t_{B 1 i} \leq 1$

(a.2) $t_{E A T i} \leq 1$

Onde: $0 \leq i \leq 24$, $i \in N$.

Assim, a restrição (a.1) garante que a bomba B1 não funcionará por mais de 60 minutos em qualquer hora do dia. Da mesma forma, a restrição (a.2) garante que a solução não indicará funcionamento de mais de 60 minutos/hora disponível. 
b) Conjunto de restrições de volumes inicial, mínimo e máximo dos reservatórios $\left(m^{3}\right)$

O volume inicial de cada reservatório é dado por $70 \%$ da sua capacidade máxima, conforme dados da Tabela 1. O volume a cada hora é dado pela soma do volume inicial do reservatório, mais o volume de entrada menos o volume de saída até a hora "n". A cada hora, os reservatórios não devem extravasar, ou seja, ultrapassar o volume máximo projetado para reservação.

Desta forma, o volume máximo do primeiro reservatório na hora "n" pode ser encontrado pela notação:

$$
\text { (b.1) } V_{R A P 020 n}=70,020+Q_{1} \cdot n-Q_{2} \cdot \sum_{i=1}^{n} t_{B 1 i} \leq 100,028 m^{3}
$$

Onde:

$V_{\text {RAP020n: }}$ Volume de água no reservatório RAP020 na hora "n"

$\mathrm{n}$ : Hora do dia (1 a 24). Termo utilizado para sumarizar as 24 equações de cada hora.

$Q_{1}$ : Vazão de entrada no reservatório RAP020

$\mathrm{Q}_{2}$ : Vazão de saída do reservatório RAP020 quando a bomba B1 está ligada

$t_{B 1 i}$ : Tempo de operação da bomba B1 na hora "i" em um dia

A restrição (b.1) na verdade representa um conjunto de restrições que garantem a limitação de volume máximo do reservatório RAP020 em cada hora do dia. Desta forma, o termo " $n$ " foi utilizado para sintetizar as 24 restrições referentes ao volume cumulativo em cada uma das 24 horas do dia.

Quanto ao volume mínimo do reservatório RAP020, será adotado um terço do volume máximo do reservatório (conforme Tabela 1), portanto:

$$
\text { (b.2) } 70,020+Q_{1} \cdot n-Q_{2} \cdot \sum_{i=1}^{n} t_{B 1 i} \geq 33,343 \mathrm{~m}^{3}
$$

Da mesma forma que para a restrição (b.1), a restrição (b.2) representa um conjunto de restrições que garantem a limitação de volume mínimo do reservatório RAP020 em cada hora do dia. Desta forma, o termo " $n$ " foi utilizado para sintetizar as 24 restrições referentes ao volume cumulativo em cada uma das 24 horas do dia. 
O volume reservado do segundo reservatório (RAP012) deve respeitar as equações seguintes, baseadas nas dimensões físicas do mesmo e vazões horárias de entrada e saída:

$$
\text { (b.3) } V_{R A P 012 n}=282,395+Q_{2} \cdot \sum_{i=1}^{n} t_{B 1 i}-Q_{3} \cdot \sum_{i=1}^{n} t_{E A T i} \leq 403,422 \mathrm{~m}^{3}
$$

Onde:

$V_{\text {RAP012n: }} \quad$ Volume de água no reservatório RAP012 na hora "n" $t_{\text {EATi }}$ : Tempo de operação da bomba EAT010 na hora "i" em um dia

$Q_{2}$ : Vazão de entrada no reservatório RAP012 quando a bomba B1 está ligada $Q_{3}$ : Vazão de saída do reservatório RAP012 quando a bomba EAT está ligada $t_{B 1 i}$ : Tempo de operação da bomba B1 na hora "i" em um dia

Assim, (b.3) representa um conjunto de restrições que garantem a limitação de volume máximo do reservatório RAP012 em cada hora do dia. Desta forma, o termo " $n$ " foi utilizado para sintetizar as 24 restrições referentes ao volume cumulativo em cada uma das 24 horas do dia.

Da mesma forma, o volume do reservatório RAP012 deve sempre ser superior a $1 / 3$ do volume máximo:

$$
\text { (b.4) } 282,395+Q_{2} \cdot \sum_{i=1}^{n} t_{B 1 i}-Q_{3} \cdot \sum_{i=1}^{n} t_{E A T i} \geq 134,474 \mathrm{~m}^{3}
$$

Assim, (b.4) representa um conjunto de 24 restrições que garantem a limitação de volume mínimo do reservatório RAP012 em cada hora do dia.

O volume reservado no terceiro reservatório (REL005) deve ser restrito pelas equações seguintes, que garantem o não-extravasamento do reservatório:

$$
\text { (b.5) } V_{R E L 005 n}=72,943+Q_{3} \cdot \sum_{i=1}^{n} t_{E A T i}-\sum_{i=1}^{n} d_{n} \leq 104,204 \mathrm{~m}^{3}
$$

Onde:

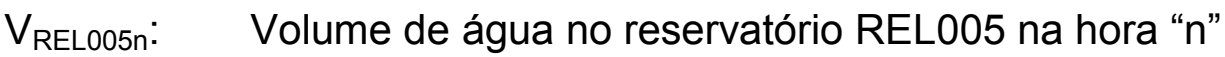
tEATi: Tempo de operação da bomba EAT010 até a hora " $n$ "

$Q_{3}$ : Vazão de entrada no reservatório REL005 quando a bomba EAT está ligada 
Q4: Vazão de saída do reservatório REL005

i : Hora do dia

$d_{n}$ : Demanda em $m^{3}$ para a hora " $n$ " (conforme valores da Tabela 3 apresentada)

Desta forma, (b.5) representa um conjunto de 24 restrições que garantem a limitação de volume máximo do reservatório REL005 em cada hora do dia.

Volume mínimo do reservatório REL005 (1/3 do volume máximo):

(b.6) $72,943+Q_{3} \cdot \sum_{i=1}^{n} t_{E A T i}-\sum_{i=1}^{n} d_{n} \geq 34,735 m^{3}$

Desta forma, (b.6) representa um conjunto de 24 restrições que garantem a limitação de volume mínimo do reservatório REL005 em cada hora do dia. O valor " $d_{n}$ " nos conjuntos de restrições (b.5) e (b.6) garante o atendimento da demanda total do dia, assim como o atendimento da demanda (consumo) horária.

c) Restrições de volume de água no início e fim do dia

O volume de água no início do período (a Oh) dos reservatórios deve ser igual ao volume no final do dia (às $24 \mathrm{~h}$ ). Isto significa que a variação de volume depois de 24 horas deve ser igual a zero, para garantir que o padrão de operação de bombas possa ser repetido em longo prazo, atendendo a demanda. Considera-se que a variação de volume do reservatório no final do dia é o volume de entrada de água do dia menos o volume de saída de água do dia, e deve ser igual a zero.

Para o reservatório RAP020:

(c.1) $Q_{1} \cdot 24-Q_{2} \cdot \sum_{i=1}^{24} t_{B 1 i}=0$

Para o reservatório REL005 (para demanda total do dia 384,048 $\mathrm{m}^{3}$ ):

(c.2) $Q_{3} \cdot \sum_{i=1}^{24} t_{E A T i}=\sum_{i=1}^{24} d_{i}=384,048$

Para o reservatório RAP012 (intermediário) não é necessária esta restrição, pois as outras restrições (c.1) e (c.2) já garantem o funcionamento do padrão de operação de bombas em longo prazo. 


\subsection{Restrições de não-negatividade}

$$
t_{B 1 i} ; t_{E A T i} \geq 0
$$

\section{RESULTADOS E ANÁLISE}

O software LINDO trouxe como solução ótima para o problema descrito e modelado no capítulo anterior:

Custo Energético $=\mathrm{R} \$ 16,18425 /$ dia de operação das bombas, o que representa um custo mensal médio de $R \$ 485,00$. Para esta solução, as variáveis tomam os valores da Tabela 4 a seguir.

Tabela 4 - Valores das variáveis do problema na solução ótima (em horas)

\begin{tabular}{|c|c|c|c|}
\hline \multicolumn{2}{|c|}{ Bomba B1 } & \multicolumn{2}{|c|}{ Bomba EAT } \\
\hline Intervalo do dia & Valor (h) & Intervalo do dia & Valor (h) \\
\hline $0 h-1 h$ & 0,0979 & $0 h-1 h$ & 0,0349 \\
\hline $1 h-2 h$ & 0,0000 & $1 h-2 h$ & 0,0000 \\
\hline $2 h-3 h$ & 0,2941 & $2 h-3 h$ & 0,0000 \\
\hline $3 h-4 h$ & 0,2941 & $3 h-4 h$ & 0,0000 \\
\hline $4 h-5 h$ & 0,2942 & $4 h-5 h$ & 0,0000 \\
\hline $5 h-6 h$ & 0,2941 & $5 h-6 h$ & 0,0000 \\
\hline $6 h-7 h$ & 0,2941 & $6 h-7 h$ & 0,0000 \\
\hline $7 h-8 h$ & 0,2941 & $7 h-8 h$ & 0,0907 \\
\hline $8 h-9 h$ & 0,2941 & $8 h-9 h$ & 0,1165 \\
\hline $9 h-10 h$ & 0,2941 & $9 h-10 h$ & 0,1316 \\
\hline $10 h-11 h$ & 0,2942 & $10 h-11 h$ & 0,1760 \\
\hline $11 \mathrm{~h}-12 \mathrm{~h}$ & 0,2941 & $11 \mathrm{~h}-12 \mathrm{~h}$ & 0,1511 \\
\hline $12 h-13 h$ & 0,2941 & $12 h-13 h$ & 0,1378 \\
\hline $13 h-14 h$ & 0,2941 & $13 h-14 h$ & 0,1351 \\
\hline $14 h-15 h$ & 0,2941 & $14 h-15 h$ & 0,1093 \\
\hline $15 h-16 h$ & 0,2942 & $15 h-16 h$ & 0,1165 \\
\hline $16 h-17 h$ & 0,4705 & $16 h-17 h$ & 0,1085 \\
\hline $17 \mathrm{~h}-18 \mathrm{~h}$ & 1,0000 & $17 \mathrm{~h}-18 \mathrm{~h}$ & 0,4249 \\
\hline $18 h-19 h$ & 0,0000 & $18 h-19 h$ & 0,0000 \\
\hline $19 h-20 h$ & 0,0000 & $19 h-20 h$ & 0,0000 \\
\hline $20 h-21 h$ & 0,0000 & $20 h-21 h$ & 0,0000 \\
\hline
\end{tabular}

Revista Produção Online, Florianópolis, SC, v.13, n. 2, p. 500-519, abr./jun. 2013. 
O padrão ótimo de operação das bombas mantém ambas desligadas nos horários de pico, uma vez que as capacidades de reservação máxima e mínima dos reservatórios são atendidas, assim como a demanda do sistema. No caso de uma redução da capacidade de reservação e/ou aumento significativo na demanda de água, poderia haver a necessidade de acionar pelo menos uma das duas bombas durante o período da tarifa de ponta para suprir a demanda, o que acarretaria em um incremento no custo de operação.

Para melhor ilustrar o funcionamento do sistema de distribuição de água, o Gráfico 2 a seguir representa a hora do dia (eixo $x$ do gráfico) e o tempo de operação de cada bomba nesta hora (eixo y) ao longo das 24 horas do dia.

Gráfico 2 - Padrão de operação das Bombas B1 e EAT.

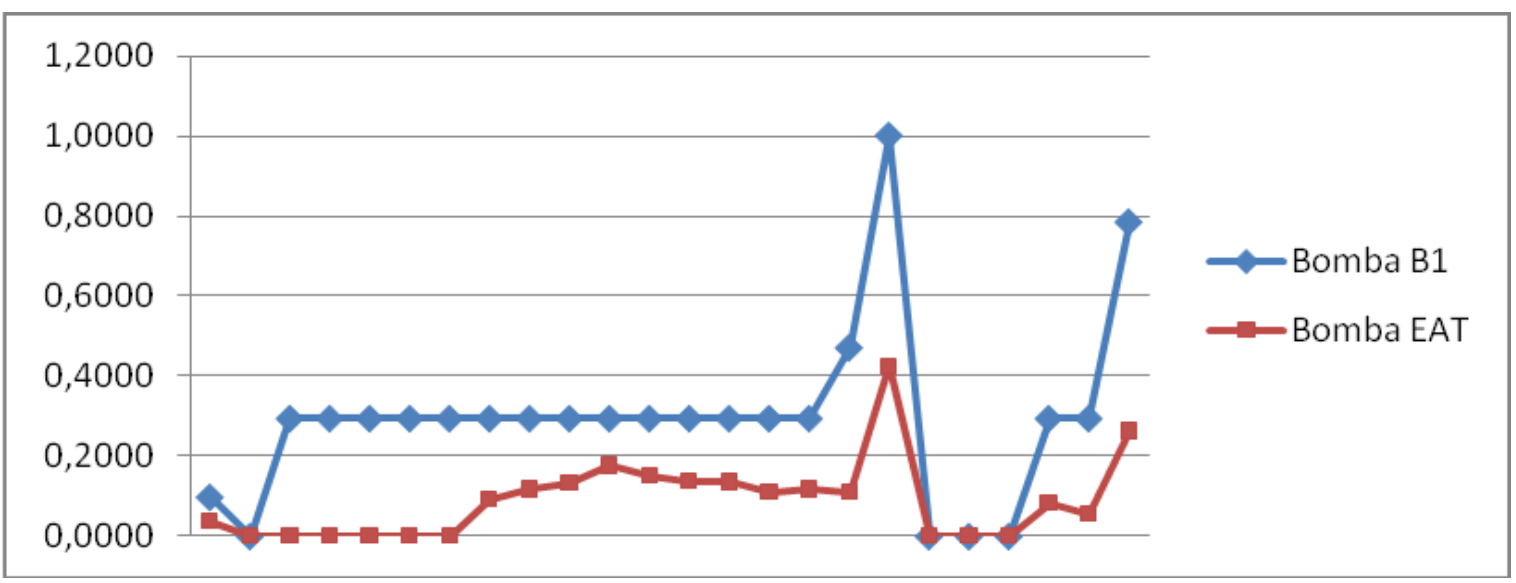

O Gráfico 2 aponta que as bombas estão, na maior parte do tempo, desligadas, sendo que a bomba B1 funciona sete horas e quatro minutos por dia e a bomba EAT funciona apenas duas horas e oito minutos. Além disso, o maior tempo de funcionamento das duas bombas por hora é no intervalo de hora anterior ao início da tarifa de ponta, para garantir que o sistema abasteça a demanda de água sem ter que acionar as bombas durante estas três horas mais caras. Nota-se também que o padrão ótimo de operação prevê que a bomba EAT fique desligada no início do dia (da $1 \mathrm{~h}$ às $7 \mathrm{~h}$ ), o que poderia ser intuitivo uma vez que a demanda neste período é bem baixa. 


\section{ANÁLISE DE PÓS-OTIMALIDADE}

A análise de pós-otimalidade permite verificar as possíveis alterações dos parâmetros do sistema, e sua influência ou não na função objetivo, ou seja, no custo energético. Primeiramente, é válido mencionar que a concessionária de energia elétrica também possui a tarifa convencional de operação, a um custo de 0,31421 $\mathrm{R} \$ / \mathrm{kWh}$, com $15 \%$ de desconto para sistemas de água e esgoto, portanto 0,267079 $\mathrm{R} \$ / \mathrm{kWh}$. Caso este mesmo sistema de distribuição operasse sob esta tarifa convencional, onde todas as horas do dia têm o mesmo custo, o CE obteria valor R $\$$ 30,63 por dia e $\mathrm{R} \$ 918,93$ por mês. Por isso, confirma-se que operar com o tarifário horo-sazonal verde é mais vantajoso financeiramente.

Quanto aos coeficientes das variáveis de decisão na função objetivo, os coeficientes referentes às horas de pico ( $i=19,20$ e 21) das duas bombas podem ser aumentados até infinito que não alteraria a solução ótima, pois os valores das variáveis continuariam iguais a zero. Isto é, mesmo que a tarifa do horário de ponta aumente, a solução ótima permanece a mesma. Quando ao decréscimo destes valores, a tarifa de horário de ponta poderia reduzir em até $R \$ 0,61 / \mathrm{kWh}$ sem alterar o padrão de funcionamento dado pela solução ótima.

Caso haja um aumento de demanda de água de $11,4 \%$, no qual a demanda passaria de 4,445 L/s para 4,95 L/s, o CE do sistema seria de $R \$ 16,77$ por dia ou $\mathrm{R} \$ 503,10$ por mês, o que representa um aumento de $\mathrm{R} \$ 0,59$ por dia. O CE aumentou somente o valor proporcional ao aumento de volume de água para a tarifa horo-sazonal fora de ponta, uma vez que as capacidades de reservação ainda permitem que as bombas não necessitem operar das 18 as $21 \mathrm{~h}$, quando a tarifa é mais cara, mesmo com este acréscimo na demanda.

\section{CONSIDERAÇÕES FINAIS}

Este trabalho apresentou um estudo de otimização sobre um sistema de distribuição de água que utiliza duas bombas com potências fixas e três reservatórios de água tratada. O objetivo foi traçar um padrão de operação ótimo para as bombas em um período de 24 horas a fim de minimizar os custos 
energéticos relativos às bombas, obedecendo às restrições de capacidade de reservação e atendimento da demanda. A metodologia utilizada para a modelagem do problema foi a Programação Linear e o software utilizado para solucionar o modelo matemático foi o LINDO.

A solução do problema atendeu o objetivo desta pesquisa uma vez que forneceu um padrão de funcionamento de cada uma das bombas em termos de tempo de bomba ligada em cada hora do dia, que poderia variar de zero até uma hora. A modelagem do problema e a análise de pós-otimalidade permitiram concluir que o sistema de distribuição está superdimensionado para a atual demanda do bairro.

Vale destacar que a modelagem não considerou os possíveis custos de manutenção associados ao religamento frequente das bombas, que influenciariam o custo de longo prazo do sistema, obtendo valores diferentes se a bomba fosse ligada e desligada uma ou 40 vezes no dia, por exemplo. Entretanto, como o modelo considera que a bomba não será religada mais do que 24 vezes em um dia, devido à estruturação do problema de forma horária, este impacto pôde ser desconsiderado. Para considerar este custo, a FO do problema teria um termo adicional agregando custo de manutenção de cada bomba em função do religamento.

Outro ponto relevante diz respeito à potência das duas bombas, que é muito superior ao que o sistema de distribuição necessita e, por este motivo, ambas permanecem a maior parte do tempo desligadas.

Este trabalho evidencia que a utilização da programação linear é satisfatória para a resolução deste tipo de problema de distribuição de água, auxiliando na busca de redução de custos energéticos. A redução de custo energético para um sistema de abastecimento de água beneficia não somente a empresa de saneamento, mas também pode beneficiar a população com menores tarifas de água e, em uma visão mais holística, a sustentabilidade ambiental, ao consumir menos energia. 


\section{REFERÊNCIAS}

COSTA, A. R. N.; SILVA, A. L. da. O planejamento do processo produtivo de uma indústria de panificação por modelos matemáticos. Revista Produção Online, v.10, n.1, p. 198-222, mar. de 2010. Disponível em:

$<$ http://www.producaoonline.org.br/index.php/rpo/article/view/228>.

CUNHA, A. A. R. Otimização energética em tempo real da operação de sistemas de abastecimento de água. 2009. 191 f. Dissertação (Mestrado) - Escola de Engenharia de São Carlos, Universidade de São Paulo, São Carlos, 2009.

DANTZIG, G. B. Linear programming and extensions. New Jersey: Princeton University Press, 1998.

FRANCATO, A. L., BARBOSA, P. S. F. Operação ótima de sistemas urbanos de abastecimento de água. In: CONGRESSO BRASILEIRO DE ENGENHARIA MECÂNICA (COBEM), 15,. 1999, Águas de Lindóia. Águas de Lindóia, 1999.

GOLDBARG, M. C., LUNA, H. P. Otimização combinatória e programação linear: modelos e algoritmos. Rio de Janeiro: Campus, 2000.

GOMES JR, A. C., SOUZA, M. J. F. Softwares de Otimização: manual de referência. Disponível em:

<http://www.decom.ufop.br/prof/marcone/Disciplinas/OtimizacaoCombinatoria/Manua IdeSoftwaresdeOtimizacao.pdf>. Acesso em: 10 jun. 2011.

KANG, D.; LANSEY, K. Revisiting optimal water distribution system design: issues and a heuristic hierarchical approach. Journal of Water Resources Planning and Management. Accepted manuscript. 2011.

PASHA, M.; LANSEY, K. Optimal pump scheduling by linear programming. In: WORLD ENVIRONMENTAL AND WATER RESOURCES CONGRESS, 2009, Kansas City.

SANEAR, PROCEL. Programa nacional de conservação de energia elétrica no setor de saneamento. Disponível em:

<http://www.eletrobras.com/elb/data/Pages/LUMISEB7EA1A1ITEMID2F33E97CD65 F4F64BDCD1F88F55C6712PTBRIE.htm>. Acesso em: 10 jun. 2011.

TSUTIYA, M. T. Redução do custo de energia elétrica de abastecimento de água. São Paulo: Associação Brasileira de Eng. Sanitária e Ambiental, 2001.

VENTURINI, M. A. A. G. Contribuição ao estudo de otimização de redes hidráulicas através de um modelo de programação linear. 1997. $111 \mathrm{f}$. Dissertação (Mestrado) - Engenharia Civil, Universidade Estadual de Campinas, Campinas, 1997. 
YANG, S.; HSU, N. S.; LOUIE, P. W. F.; YEH, W. W. G. Water distribution network reliability: connectivity analysis. Journal of Infrastructure Systems, v.2, n.2, p.5464. 1996.

ZECCHIN, A.C. Application of two ant colony optimisation algorithms to water distribution system optimization. Mathematical and Computer Modelling, v.44, n.56, p.451-468. 2006.

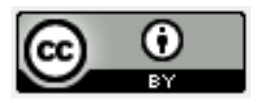

Artigo recebido em 06/10/2011 e aceito para publicação em 24/01/2012. 